\title{
High Depth Resolution Auger Depth Profiling Analysis Using Inclined Holder
}

\author{
T. Ogiwara*, T. Nagatomi** and S. Tanuma*
}

*Materials Analysis Station, National Institute for Materials Science

1-2-1 Sengen, Tsukuba-shi, Ibaraki 305-0047, Japan

**Graduate School of Engineering, Osaka University

1-1 Yamadaoka, Suita, Osaka 565-0871, Japan

Auger electron spectroscopy (AES) combined with argon ion sputtering is widely used for the depth profiling of multilayer structures. In this method, it is very important to measure the depth profile of a specimen with high depth resolution to evaluate the structure of materials precisely. In this work, we have carried out the high depth resolution Auger depth profiling analysis with a very shallow incident angle of argon ion for sputtering using an inclined holder.

A schematic view of the shallow angle argon ion sputtering method using an inclined holder is shown in Fig.1. The sample is mounted on a slanted sample holder. The sample surface is inclined over an angle $45^{\circ}$ with respect to the base plane. In consequence, the angle between the sample surface normal and the electron beam direction is $45^{\circ}$ with independence of the azimuthal rotation [1]. At the same time, the incidence angle of argon ions can be varied from $38.9^{\circ}$ to $83.3^{\circ}$ by rotating the sample. In this way, the inclined holder enables the very shallow incident angle of argon ion for sputtering to be used. In order to examine efficiency of this method, we have performed Auger depth profiling of a GaAs/AlAs multilayer specimen using the inclined holder.

The measured Auger depth profiles of the GaAs/AlAs multilayer specimen using the inclined holder are shown in Fig. 2(a). The incidence angle of argon ions was $83.3^{\circ}$. For comparison, the Auger depth profile measured by the conventional method at the incidence angle of $38.9^{\circ}$ is shown in Fig 2(b). The depth resolutions of Ga LMM and Al KLL obtained from Figs. 2(a) and (b) versus the depth from the surfaces are summarized in Fig. 3. The depth resolution is defined by the distance between $16 \%$ and $84 \%$ (or $84 \%$ and $16 \%$ ) of the intensity change at an interface. The depth profile of Ga-LMM and AlKLL in Fig. 2(a) showed much better the depth resolution compared to that of the conventional method as shown in Fig. 2(b). We applied the inclined holder to the measurement of a Si/Ge multiple delta-layer specimen. The measured Ge LMM Auger depth profile of the Si/Ge multiple delta-layer specimen is shown in Fig. 4. We can observe the Ge mono-layer of the $\mathrm{Si} / \mathrm{Ge}$ multiple delta-layer specimen using an inclined holder. This method is the practical measurement method utilizing the characteristic of the hemisphere type AES device.

[1] W. S. M. Werner, W. Smekal and H. Stori, Surf. Interface Anal. 31, 475 (2001). 


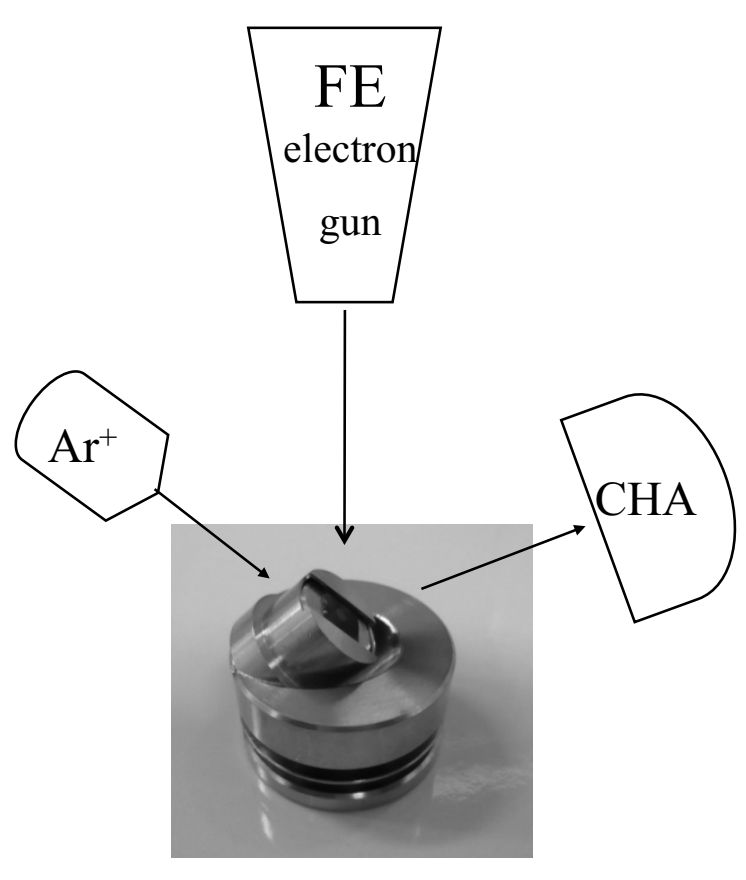

Fig. 1. Schematic view of the shallow incident angle ion sputtering method using the inclined holder.

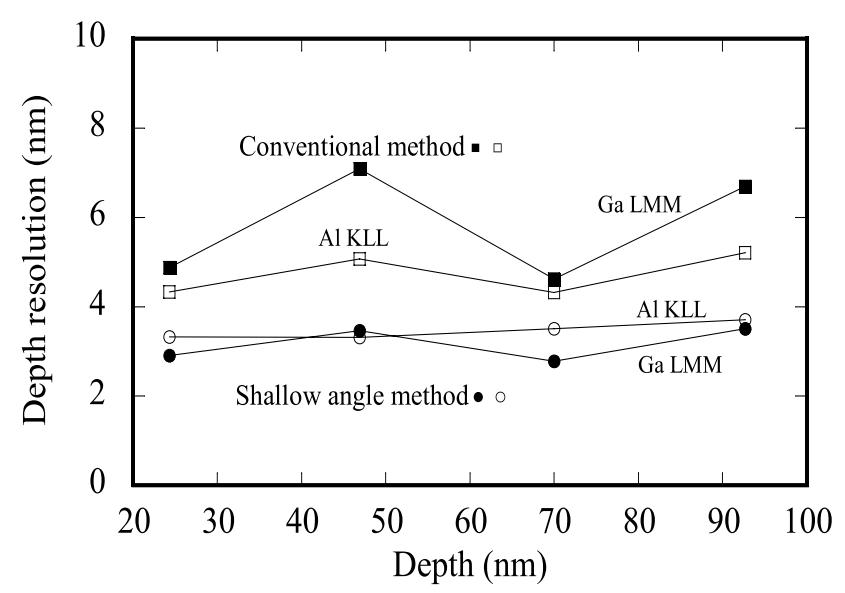

Fig. 3. Dependence of the depth resolution of the GaAs/AlAs multilayer specimen on the depth from the surface.
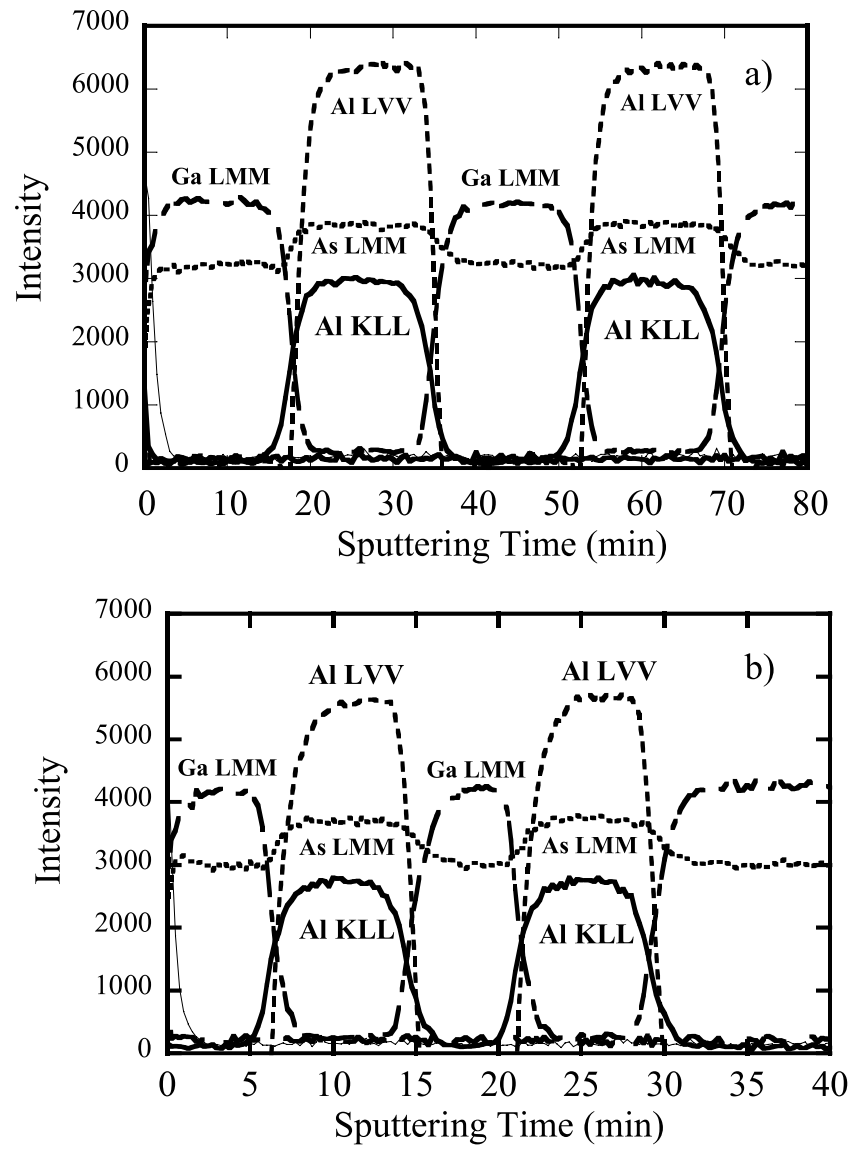

Fig. 2. AES depth profiles of the GaAs/AlAs multilayer specimen obtained using (a) the inclined holder and (b) conventional method.

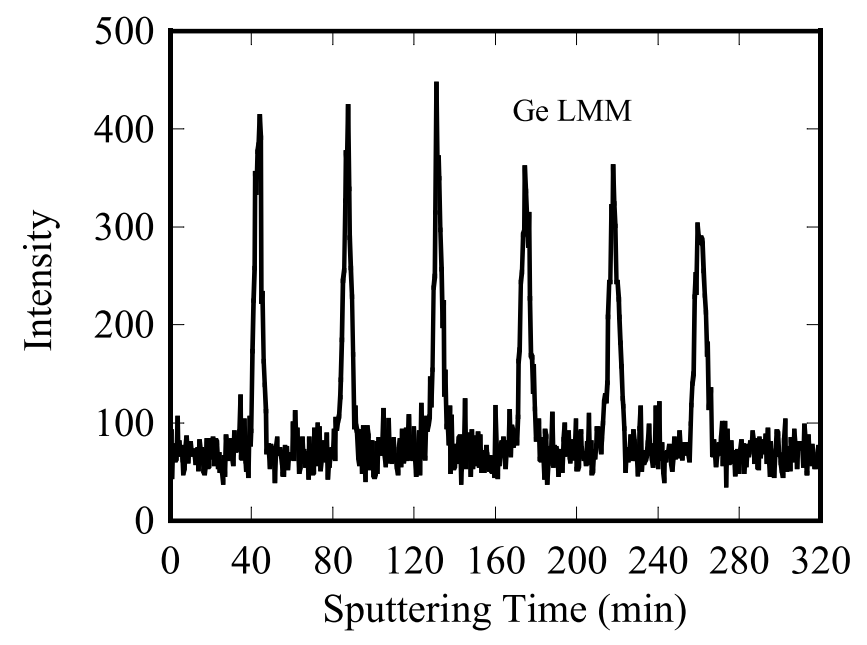

Fig. 4. Ge LMM AES depth profile of the Si/ Ge multiple delta-layer specimen using an inclined holder. 